\title{
Condición Periodontal de los Dientes Temporales Adyacentes a la Hendidura Alveolar en Pacientes con Labio y Paladar Hendido Unilateral No Sindrómico, Hospital Nacional de Niños "Dr. Carlos Sáenz Herrera", Caja Costarricense de Seguro Social
}

\author{
Periodontal Condition of Primary Teeth Adjacent to the Alveolar Cleft in Patients with \\ Non-Syndromic Unilateral Cleft Lip and Palate, Hospital Nacional de Niños "Dr. Carlos \\ Sáenz Herrera", Caja Costarricense de Seguro Social
}

María del Carmen Navas-Aparicio ${ }^{1,2}$ \& María Alejandra Vargas-Mora ${ }^{3}$

NAVAS-APARICIO, M. C. \& VARGAS-MORA, M. A. Condición periodontal de los dientes temporales adyacentes a la hendidura alveolar en pacientes con labio y paladar hendido unilateral no sindrómico, Hospital Nacional de Niños "Dr. Carlos Sáenz Herrera", Caja Costarricense de Seguro Social. Int. J. Odontostomat., 15(4):1009-1018, 2021.

RESUMEN: Los dientes adyacentes a la hendidura alveolar, en pacientes con labio y paladar hendido, presentan mayor incidencia de problemas periodontales, según la literatura. El objetivo de este trabajo fue evaluar si los dientes temporales adyacentes a la hendidura alveolar presentan mayor incidencia de problemas periodontales en niños con labio y paladar hendido unilateral completo no sindrómico, nacidos en 2010 y 2011, atendidos en el Hospital Nacional de Niños "Dr. Carlos Sáenz Herrera", Caja Costarricense de Seguro Social. Este estudio prospectivo, transversal, descriptivo, e intervencional evaluó variables epidemiológicas, clínicas y radiográficas (placa bacteriana, profundidad de bolsa, nivel de inserción, recesión gingival, sangrado, encía queratinizada e insertada, cálculo y movilidad dental, tipo de hendidura alveolar). Se utilizó técnicas de análisis estadísticos descriptivas (distribución de frecuencias, cruce de variables y construcción de intervalos de confianza).El análisis de datos y procesamiento estadístico se realizó en SPSS versión 17.0 y en Excel. El grupo constó de 17 pacientes, con edad promedio de 5 años. Se encontró $47 \%$ de placa dental y $0 \%$ de cálculo dental. La profundidad de bolsa y el sangrado fueron estadísticamente mayores para los dientes dentro de la hendidura alveolar. Los dientes adyacentes a la hendidura presentaron menor cantidad de encía queratinizada e insertada. Referente a la movilidad dental no existe diferencia estadísticamente significativa. Solo un diente presentó recesión gingival. La hendidura alveolar completa predominó radiográficamente. Los dientes cercanos a la hendidura alveolar tienen mayor predisposición a problemas periodontales, por lo que es relevante el seguimiento regular de la salud periodontal. gingivitis.

PALABRAS CLAVE: hendidura alveolar, dientes temporales, profundidad de bolsa, nivel de inserción clínica, periodontitis,

\section{INTRODUCCIÓN}

En la literatura se reporta que los dientes adyacentes a la hendidura alveolar,en pacientes con labio y paladar hendido, presentan una mayor incidencia de problemas periodontales que el resto de los dientes en el arco dental, por la relación existen- te con el defecto óseo (Schultes et al.,1999; Costa et al., 2003), el tejido cicatrizal producto de las cirugías(Costa et al.; Uddin et al., 2007), la malposición y maloclusión dental tanto en la dentición temporal como permanente (Costa et al.; Uddin

\footnotetext{
${ }^{1}$ Hospital Nacional de Niños Carlos Sáenz Herrera, Caja Costarricense de Seguro Social. Costa Rica, 1654-1000, San José Costa Rica.

${ }^{2}$ Facultad de Odontología, Universidad de Costa Rica,San José, Costa Rica.

${ }^{3}$ Facultad Autónoma de Ciencias Odontológicas Heredia, Costa Rica.
} 
et al.; Stec et al., 2007; Gamba Garib et al., 2012), así como por los dientes que tienen una malformación en su desarrollo,entre otros factores, lo cual trae como consecuencia dificultad en la buena higiene oral, además como acúmulo de placa bacteriana (Uddin et al.) y predisposición a la aparición de patologías gingivales y periodontales en esta zona (Stec et al.).

Sobre la condición periodontal de los dientes que erupcionan en la hendidura alveolar, al mismo tiempo como de los dientes adyacentes a esta, existen reportes que no encontraron diferencias al compararlos con sus contralaterales, mientras que otros refieren que sí presentan una mayor afectación.

Bragger et al. (1985), encontraron que radiográficamente la altura de la cresta ósea es significativamente menor en los sitios adyacentes a la hendidura alveolar sin que esto se reflejara clínicamente pues el nivel de inserción se mantenía similar tanto en dientes adyacentes a la hendidura como en sus contralaterales. Salvi et al. (2003), confirmaron este hallazgo, al realizar control periodontal a aquellos pacientes estudiados anteriormente, después de 14 años.

Boloor \& Thomas (2010) establecieron que los pacientes con hendiduras alveolares son más propensos a sufrir enfermedades periodontales debido a que acumulan una mayor cantidad de alimentos en la zona del defecto (hendidura) y se ven limitados para mantener buena higiene oral. Además, señalan que la severidad de la enfermedad periodontal es mayor si el defecto es grande e involucra el labio, el paladar y el reborde alveolar. Esta aseveración coincide con el estudio de Huynh-Ba et al. (2009).

Stec et al. estudiaron el estado periodontal y la higiene oral en pacientes entre 3.5 y 18 años de edad con hendiduras alveolares. Encontraron que todos ellos presentaban una higiene oral inadecuada, y la mayoría tenían bolsas periodontales mayores a $3.5 \mathrm{~mm}$ y recesiones gingivales, esto debido a la frecuente malposición y malformación dental en los dientes cercanos a las hendiduras alveolares.

El objetivo de este estudio fue determinar si los dientes temporales adyacentes a la hendidura alveolar presentan mayor incidencia de problemas periodontales en comparación con los contralaterales, en niños con labio y paladar hendido unilateral completo no sindrómico.

\section{MATERIAL Y MÉTODO}

Con la respectiva aprobación por parte del Comité Local de Bioética e Investigación del Hospital Nacional de Niños, Caja Costarricense de Seguro Social, CLOBI-HNN-012-2015 y del Comité Ético Científico de la Universidad de Costa Rica, B5344, se llevó a cabo este estudio, el cual es de carácter prospectivo, transversal, descriptivo e intervencional, ya que se basó en datos actuales clínicos y radiográficos (radiografía periapical de la hendidura alveolar) del paciente, para la evaluación de la condición periodontal de los dientes temporales adyacentes a la hendidura, en niños con labio paladar hendido no sindrómico completo unilateral, nacidos en el año 2010 y 2011, y atendidos en el Hospital Nacional de Niños "Dr. Carlos Sáenz Herrera", Caja Costarricense de Seguro Social.

La población de estudio consistió en 38 sujetos nacidos en el año 2010 y 2011, que cumpliesen con los siguientes criterios de inclusión: hendidura unilateral completa no sindrómica, tener 5 años de edad en el momento del estudio, dentición temporal, no haber iniciado el tratamiento de ortodoncia, no haber recibido la cirugía de injerto óseo en la hendidura alveolar y poseer la autorización respectiva para participar en el estudio.

Al incluir todos aquellos niños que cumpliesen con los criterios de inclusión, se eliminó el sesgo de selección y por tanto el error de muestreo. Entre los criterios de exclusión se encontraban: hendidura labial, alveolar y/o palatina incompleta y/o bilateral, tratamiento de ortodoncia iniciado, cirugía realizada de injerto óseo en la hendidura alveolar, pacientes poco cooperadores, datos incompletos en el expediente y radiografías inadecuadas. Además, no se analizaron dientes con fractura radicular, con tratamiento de conducto o lesión periapical, con movilidad generada por trauma inmediato o reabsorción radicular patológica y con destrucción coronal subgingival, con coronas de acero cromado desadaptadas o restauraciones subgingivales que impidiesen la identificación del límite amelo-cemento, promovieran la retención de placa bacteriana y limitaran el acceso a la higiene.

El levantamiento de datos se realizó a través de dos examinadoras previamente calibradas.

El instrumental utilizado consistió de espejo intraoral \#5, sonda periodontal Carolina del Norte (milímetro a milímetro), jeringa triple, rodillos de algodón, 
NAVAS-APARICIO, M. C. \& VARGAS-MORA, M. A. Condición periodontal de los dientes temporales adyacentes a la hendidura alveolar en pacientes con labio y paladar hendido unilateral no sindrómico, Hospital Nacional de Niños "Dr. Carlos Sáenz Herrera”, Caja Costarricense de Seguro Social. Int. J. Odontostomat., 15(4):1009-1018, 2021.

separadores y espejos de rodio para la toma de fotografías y posicionadores para la toma de radiografías. Para la compilación de la información se utilizó una hoja de recolección de datos que incluyó variables epidemiológicas (sexo, lugar de procedencia, edad actual) y las siguientes variables clínicas y radiográficas:

Placa bacteriana: masa organizada, consistente principalmente de microorganismos, con capacidad de adherirse a dientes, prótesis dentales, y tejidos orales, incluyendo el surco gingival y bolsas periodontales (American Academy of Periodontology, 2001). Su medición se realizó mediante el índice de Silness y Loe (Newman et al., 2002) según se detalla:

0 : Ausencia de placa

1. Placa en el margen detectable solamente al pasar el explorador

2.: Placa visible a simple vista en el margen, espacios interproximales libres

3.: Placa visible a simple vista, presente incluso en los espacios interproximales

Cálculo Dental: concreción dura que se forma en los dientes o prótesis mediante la calcificación de la placa bacteriana. Se clasificó en supragingival (ubicado coronal al margen gingival) mediante inspección visual, y subgingival (ubicado apical al margen gingival) mediante examen táctil con la sonda periodontal.

Profundidad de bolsa: distancia desde el margen de tejido blando (encía o mucosa alveolar) a la punta de la sonda periodontal durante el sondaje. Se registró en las superficies mesial, medial y distal, en vestibular y palatino de cada diente para un total de 6 mediciones por diente.

Nivel de inserción: distancia de la unión amelo-cemento al fondo del surco.Se registró en las superficies mesial, medial y distal tanto por vestibular como por palatino, para un total de 6 mediciones por diente.

Recesión gingival: localización del margen gingival apical a la unión amelo-cemento (UAC).Se registró en sitios mediales de los dientes, en vestibular y palatino.

Sangrado al sondaje: sangrado generado al introducir la sonda periodontal en el surco para medir su profundidad.Se registró un punto sangrante por cada medición de "Profundidad de bolsa".

Encía queratinizada: Tejido fibroso cubierto por epitelio queratinizado que rodea al diente y que se ex- tiende desde el margen gingival hasta la línea mucogingival.

Ancho de la banda de encía insertada: porción de la encía firme y fuertemente adherida al diente que se extiende desde el fondo del surco gingival hasta la línea mucogingival (American Academy of Periodontology).

Movilidad dental: Movilidad de un diente dentro del alvéolo (Lindhe et al., 2008). Se registró utilizando el mango de dos instrumentos y se clasificó según Miller (1950) de la siguiente manera:

Grado I: movilidad aumentada a máximo $1 \mathrm{~mm}$ en sentido horizontal

Grado II: movilidad horizontal que excede $1 \mathrm{~mm}$

Grado III: movilidad severa tanto en sentido horizontal como vertical (intrusión)

Es importante considerar que,la movilidad de los dientes temporales puede ser debida a reabsorción radicular ante la erupción del diente permanente sucedáneo.

\section{Hendidura alveolar:}

Completa: abarca la separación completa de los rebordes alveolares.

Incompleta: existe un puente de hueso uniendo los rebordes alveolares adyacentes a la hendidura alveolar.

Cada variable del estudio se analizó por grupo de dientes en relación con la hendidura alveolar:

- Dientes adyacentes a la hendidura alveolar: dientes contiguos a la hendidura sea en mesial o distal.

- Dientes no adyacentes a la hendidura alveolar: dientes que no se localizan contiguo a la hendidura alveolar.

- Dientes dentro de la hendidura alveolar: dientes localizados dentro de la hendidura alveolar.

Las técnicas de análisis estadísticos fueron descriptivas, tal como distribución de frecuencias, cruce de variables y de construcción de intervalos de confianza. El análisis de datos y el procesamiento estadístico se realizó en SPSS versión 17.0 y en Excel, con el fin de probar la hipótesis de si los dientes temporales adyacentes a la hendidura alveolar son más propensos a desarrollar enfermedad periodontal. 


\section{RESULTADOS}

El grupo de trabajo constó de 17 sujetos de estudio con una edad promedio de 5 años, 11 de sexo masculino (64\%) y 6 de sexo femenino (36\%), quienes presentaban malformación de labio paladar hendido completo unilateral no sindrómico, siendo el $71 \%(n=12)$ del lado izquierdo y el $29 \%$ de lado derecho $(n=5)$.

Se estudió un total de 82 dientes de los cuales 75 fueron valorables en todas las variables previamente señaladas. El diente canino superior temporal izquierdo (6.3) es el diente que más se presentaba adyacente a la hendidura alveolar y los dientes incisivos laterales, fueron los dientes que principalmente se ubicaban en la hendidura (Fig. 1).

No se encontró diferencia estadísticamente significativa entre dientes, no adyacentes o dentro de la hendidura alveolar para la variable de movilidad $(p=0,064)$.

El promedio de encía queratinizada por diente fue de 4,1 mm (IC95\%: 3,8-4,4), encontrándose una diferencia estadísticamente significativa entre el grupo de dientes adyacentes y no adyacentes a la hendidura alveolar, siendo mayor en las no adyacentes $(p=0,001)$. Específicamente por tipo de diente no se encontró diferencia estadísticamente significativa $(p=0,292)$.
Tabla I. Ancho de banda de encía insertada por grupo de dientes.

\begin{tabular}{lccc}
\hline $\begin{array}{l}\text { Relación con la } \\
\text { hendidura }\end{array}$ & $\mathrm{N}$ & \multicolumn{2}{c}{ Conjuntos * $^{*}$} \\
\hline Hendidura & 3 & 1,33 & 2 \\
Adyacente & 27 & 2,24 & 2,24 \\
No adyacente & 45 & & 3,33 \\
Significancia & & 0,11 & 0,05
\end{tabular}

Fuente: Hospital Nacional de Niños, Caja Costarricense de Seguro Social, 2015-2016.

*Conjunto: grupos con tendencia de comportamiento estadístico similar. En este caso el grupo 1 corresponde al grupo de dientes en la hendidura y adyacentes a la hendidura, y el grupo 2 corresponde a los dientes no adyacentes a la hendidura.

Los valores para el ancho de la banda de encía insertada siguieron el mismo patrón de la encía queratinizada, siendo estadísticamente mayor en los dientes no adyacentes a la hendidura alveolar versus los adyacentes $(p=0,0001)$, además, no hubo diferencia estadísticamente significativa por tipo de diente $(p=0,955)$. El valor promedio de encía insertada fue de 2,9 mm (IC95 \%: 2,6 mm - 3,1 mm), observándose mayor variabilidad en sus valores para los dientes incisivo lateral superior izquierdo temporal (6.2) y canino superior izquierdo temporal (6.3), (Tabla I).

Del total de dientes analizados, solamente uno presentaba recesión gingival. Este diente fue un canino temporal izquierdo (6.3) perteneciente al grupo de dientes adyacentes a la hendidura alveolar. La recesión fue de $1 \mathrm{~mm}$ y se ubicaba por vestibular.

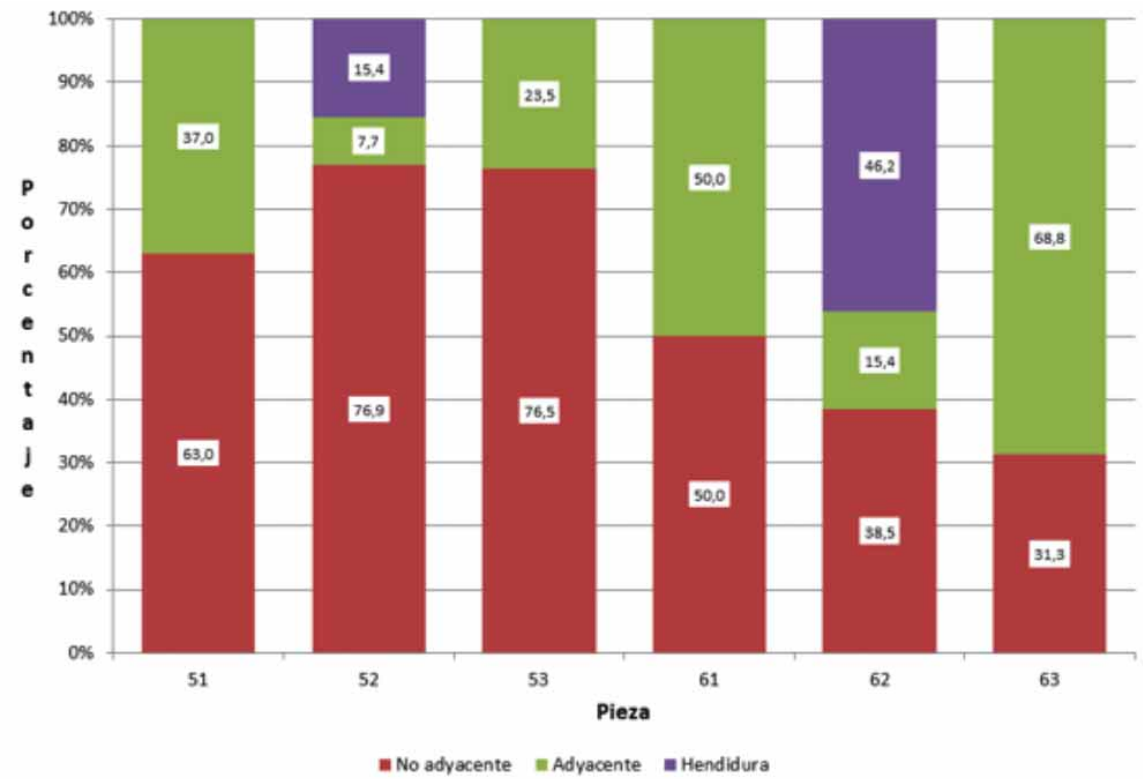

Fig. 1. Distribución de dientes según posición en la hendidura, Hospital Nacional de Niños: 2010-2011.
El $47 \%$ de las superficies dentales evaluadas presentaban placa dental siendo significativamente más alta $(p=0,001)$ en superficies vestibulares $(70 \%)$ que palatinas $(30 \%)$. Los dientes que registraron una mayor acumulación de placa fueron los que se encontraban dentro de la hendidura alveolar, siendo esta diferencia estadísticamente significativa $(p=0,04)$ con respecto al grupo de dientes adyacentes y no adyacentes a la hendidura. No se encontró diferencia estadísticamente significativa para la acumulación de placa por tipo de diente, (Tabla II). 
NAVAS-APARICIO, M. C. \& VARGAS-MORA, M. A. Condición periodontal de los dientes temporales adyacentes a la hendidura alveolar en pacientes con labio y paladar hendido unilateral no sindrómico, Hospital Nacional de Niños "Dr. Carlos Sáenz Herrera”, Caja Costarricense de Seguro Social. Int. J. Odontostomat., 15(4):1009-1018, 2021.

Tabla II. Acumulación de placa según relación del diente con la hendidura.

\begin{tabular}{lcccccccc}
\hline \multicolumn{1}{c}{ Relación con la hendidura } \\
\hline Placa & No adyacente & \multicolumn{2}{c}{ Adyacente } & \multicolumn{2}{c}{ Hendidura } & \multicolumn{2}{c}{ Total } \\
\hline 0 & $\mathrm{~N}$ & $\%$ & $\mathrm{~N}$ & $\%$ & $\mathrm{~N}$ & $\%$ & $\mathrm{~N}$ & $\%$ \\
1 & 51 & 57,3 & 27 & 49,1 & 5 & 38,5 & 83 & 52,9 \\
2 & 29 & 32,6 & 17 & 30,9 & 3 & 23,1 & 49 & 31,2 \\
3 & 7 & 7,9 & 9 & 16,4 & 3 & 23,1 & 19 & 12,1 \\
\hline Total & 2 & 2,2 & 2 & 3,6 & 2 & 15,4 & 6 & 3,8 \\
\hline
\end{tabular}

Fuente: Hospital Nacional de Niños, Caja Costarricense de Seguro Social, 2015-2016

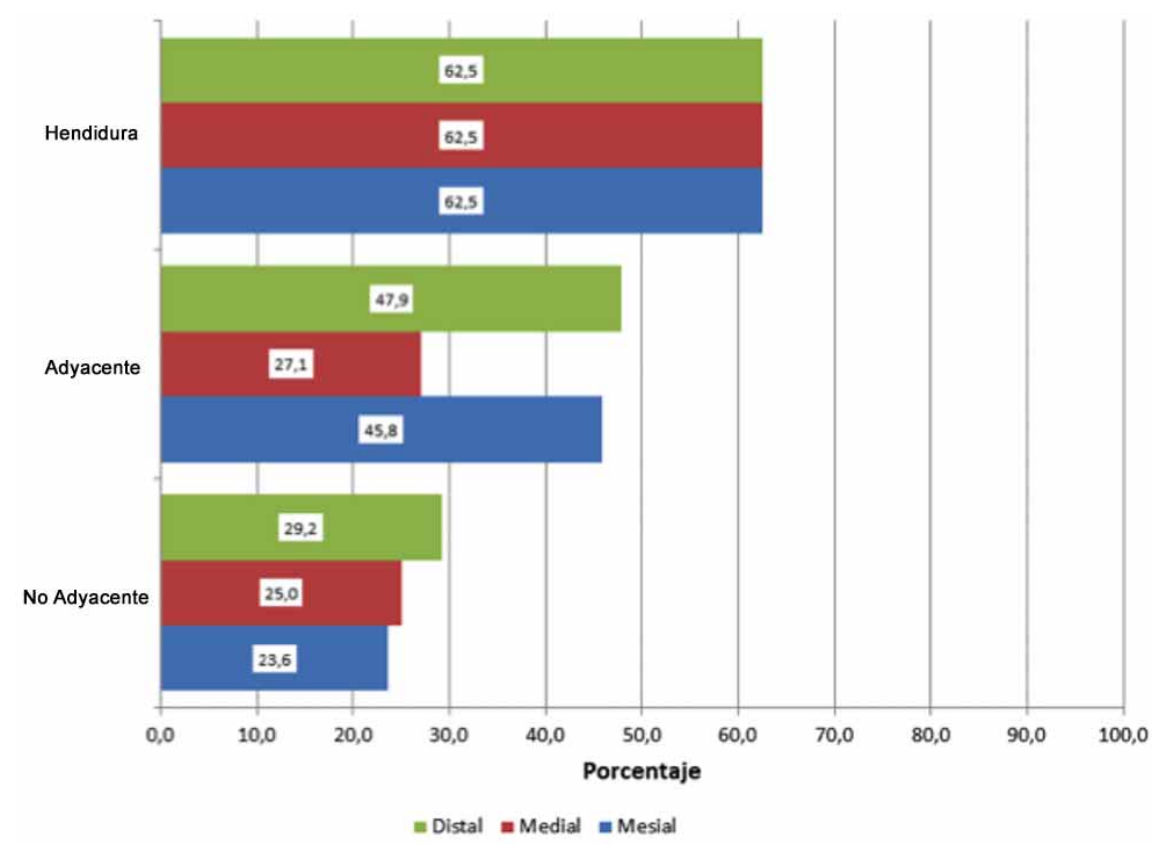

Fig. 2. Porcentaje de sangrado según relación con la hendidura y superficie dental.

No se encontró cálculo dental en ninguno de los dientes analizados.

Los dientes que registraron una mayor profundidad de bolsa fueron los que se encontraban en la hendidura. La diferencia con respecto a los otros dos grupos fue estadísticamente significativa para las superficies mesial $(p=0,001)$ y medial $(p=0,001)$, no así para la superficie distal $(p=0,352)$, (Tabla III).

El grupo de dientes adyacentes a la hendidura alveolar presentó una mayor profundidad de bolsa en sus tres superficies en comparación a los dientes no adyacentes a la hendidura, siendo esta diferencia estadísticamente significativa (mesial $p=0,0001$; medial $p=0,001$, y distal $p=0,001$ ).Según tipo de diente, no se encontró diferencia estadísticamente significativa en ninguna de sus superficies (mesial $p=0,618$; medial $p=0,05$ y distal $p=0,085$ ).
El porcentaje de sangrado fue mayor en los dientes ubicados en la hendidura, seguidos por los dientes adyacentes y dientes no adyacentes; sin embargo, se encontró diferencia estadísticamente significativa solamente al comparar los dientes dentro de la hendidura versus los dientes no adyacentes a la hendidura en sus superficies mesial $(p=0,01)$ y distal $(p=0,04)$, no así en la superficie medial $(p=0,082)$, (Fig. 2). Según tipo de diente, no se encontró diferencia estadística en ninguna de sus superficies (mesial $p=0,698$; medial $p=0,05$ y distal $p=0,582$ ).

Para el total de dientes analizados, se registraron pocas superficies con pérdida de inserción clínica (9\% de las superficies mesiales, $6 \%$ de las superficies mediales y $4 \%$ de las superficies distales). Los dientes dentro de la hendidura alveolar presentaron una mayor pérdida de inserción clínica que los dientes adyacentes y no adyacentes a la hendidura, siendo esta diferencia 
NAVAS-APARICIO, M. C. \& VARGAS-MORA, M. A. Condición periodontal de los dientes temporales adyacentes a la hendidura alveolar en pacientes con labio y paladar hendido unilateral no sindrómico, Hospital Nacional de Niños "Dr. Carlos Sáenz Herrera”, Caja Costarricense de Seguro Social. Int. J. Odontostomat., 15(4):1009-1018, 2021.

Tabla III. Profundidad de bolsa promedio según diente y superficie con relación a la hendidura.

\begin{tabular}{|c|c|c|c|c|c|c|c|c|c|c|c|c|}
\hline $\begin{array}{l}\text { Posición } \\
\text { en } \\
\text { cavidad } \\
\text { oral }\end{array}$ & & Mesia & & & & Media & & & & Dista & & \\
\hline Pieza & $\begin{array}{l}\text { No } \\
\text { adyacente }\end{array}$ & Adyacente & Hendidura & Total & $\begin{array}{l}\text { No } \\
\text { adyacente }\end{array}$ & Adyacente & Hendidura & Total & $\begin{array}{l}\text { No } \\
\text { adyacente }\end{array}$ & Adyacente & Hendidura & Total \\
\hline 51 & 1,7 & 1,8 & & 1,7 & 1,4 & 1,6 & & 1,5 & 2,1 & 1,6 & & 1,9 \\
\hline 52 & 1,8 & 5,0 & 2,5 & 2,2 & 1,1 & 2,0 & 5,0 & 1,8 & 1,6 & 2,0 & 3,0 & 1,8 \\
\hline 53 & 1,8 & 2,5 & & 1,9 & 1,0 & 1,3 & & 1,1 & 2,1 & 1,5 & & 1,9 \\
\hline 61 & 1,6 & 2,0 & & 1,8 & 1,2 & 1,0 & & 1,1 & 2,0 & 1,8 & & 1,9 \\
\hline 62 & 1,8 & 3,0 & 2,2 & 2,2 & 1,0 & 7,5 & 2,8 & 2,8 & 1,8 & 2,0 & 2,4 & 2,1 \\
\hline 63 & 1,6 & 2,0 & & 1,9 & 1,0 & 1,4 & & 1,3 & 2,0 & 1,9 & & 1,9 \\
\hline Vestibular & 1,7 & 2,2 & 2,3 & 1,9 & 1,1 & 1,8 & 3,4 & 1,6 & 1,9 & 1,8 & 2,6 & 1,9 \\
\hline 51 & 1,4 & 1,8 & & 1,5 & 1,4 & 1,6 & & 1,5 & 2,0 & 1,6 & & 1,8 \\
\hline 52 & 1,6 & 2,0 & 3,5 & 1,9 & 1,4 & 11,0 & 11,5 & 3,7 & 1,7 & 3,0 & 1,0 & 1,7 \\
\hline 53 & 1,8 & 1,5 & & 1,8 & 1,3 & 1,0 & & 1,3 & 2,0 & 1,3 & & 1,8 \\
\hline 61 & 1,6 & 2,2 & & 1,9 & 1,0 & 2,0 & & 1,5 & 1,0 & 4,2 & & 2,6 \\
\hline 62 & 1,6 & 6,0 & 6,4 & 4,3 & 1,2 & 3,0 & 4,6 & 2,9 & 1,2 & 1,5 & 4,6 & 2,7 \\
\hline 63 & 1,4 & 1,7 & & 1,6 & 1,2 & 1,7 & & 1,6 & 1,6 & 1,8 & & 1,8 \\
\hline Palatino & 1,6 & 2,1 & 5,6 & 2,1 & 1,3 & 2,1 & 6,6 & 2,0 & 1,7 & 2,1 & 3,6 & 2,0 \\
\hline 51 & 1,5 & 1,8 & & 1,6 & 1,4 & 1,6 & & 1,5 & 2,1 & 1,6 & & 1,9 \\
\hline 52 & 1,7 & 3,5 & 3,0 & 2,0 & 1,3 & 6,5 & 8,3 & 2,7 & 1,7 & 2,5 & 2,0 & 1,8 \\
\hline 53 & 1,8 & 2,0 & & 1,8 & 1,2 & 1,1 & & 1,2 & 2,0 & 1,4 & & 1,9 \\
\hline 61 & 1,6 & 2,1 & & 1,9 & 1,1 & 1,5 & & 1,3 & 1,5 & 3,0 & & 2,3 \\
\hline 62 & 1,7 & 4,5 & 4,3 & 3,3 & 1,1 & 5,3 & 3,7 & 2,9 & 1,5 & 1,8 & 3,5 & 2,4 \\
\hline 63 & 1,5 & 1,9 & & 1,8 & 1,1 & 1,5 & & 1,4 & 1,8 & 1,9 & & 1,8 \\
\hline Total & 1,7 & 2,2 & 3,9 & 2,0 & 1,2 & 1,9 & 5,0 & 1,8 & 1,8 & 2,0 & 3,1 & 2,0 \\
\hline
\end{tabular}

Fuente: Hospital Nacional de Niños, Caja Costarricense de Seguro Social, 2015-2016

estadísticamente significativa para sus superficies mesiales $(p=0,001)$, no así en medial $(p=0,311)$ y en distal $(p=0,184)$. Al comparar el grupo de dientes adyacentes con el grupo de dientes no adyacentes a la hendidura, no existió diferencia estadísticamente significativa en cuanto a los niveles de pérdida de inserción clínica.

Todos los niños del grupo bajo estudio tuvieron en común las cirugías de plastía labial primaria y plastía de paladar primaria, no así otras cirugías, según expediente digital.

En las radiografías periapicales del grupo de estudio se observó que nueve pacientes presentaban una hendidura alveolar completa. En siete niños no fue posible valorar la hendidura debido a la posición de los dientes adyacentes a esta o erupcionados en la hendidura dicha (6 sujetos), o por la angulación de la radiografía (1 sujeto). Además, hubo un niño a quien no fue posible tomarle radiografía. Cabe señalar que, aunque un criterio de exclusión era el paciente poco coopera- dor, los datos clínicos del paciente se incluyeron, debido a la importancia de los hallazgos en el grupo. La altura de la cresta alveolar no se determinó, por no poder utilizar una técnica estándar ante la falta de completa cooperación del niño.

\section{DISCUSIÓN}

La población de estudio constaba inicialmente de 38 sujetos nacidos entre el 2010 y 2011 con labio y paladar hendido unilateral no sindrómico; sin embargo, este número se redujo a 17 por razones como: imposibilidad de localización del paciente, razones laborales de los padres o encargados, falta de disponibilidad para asistir a la cita, dificultad de traslado al hospital, y/o enfermedad de los niños.

De las 17 personas del grupo de estudio,el $64 \%$ eran de sexo masculino, lo cual coincide con el estudio de Navas-Aparicio (2012), aunque existe literatura que 
NAVAS-APARICIO, M. C. \& VARGAS-MORA, M. A. Condición periodontal de los dientes temporales adyacentes a la hendidura alveolar en pacientes con labio y paladar hendido unilateral no sindrómico, Hospital Nacional de Niños "Dr. Carlos Sáenz Herrera”, Caja Costarricense de Seguro Social. Int. J. Odontostomat., 15(4):1009-1018, 2021.

señala que no existe prevalencia de sexo con respecto a la distribución de esta malformación (Aizpurua, 2002).

La edad promedio de los pacientes era de 60 meses, es decir 5 años, edad en que no ha iniciado la etapa de recambio dental, lo que permitió analizar la condición periodontal únicamente de dientes temporales con relación a la hendidura alveolar.

En este estudio se destaca que muchos de los niños presentaban ausencia de uno ó más dientes temporales del sector ántero-superior, lo cual redujo la cantidad de dientes analizados. Esta ausencia podría deberse a la pérdida temprana asociada a caries dental extensa no restaurable por una inadecuada higiene oral, a agenesia de algún diente cercano a la hendidura alveolar o a extracción previa del diente localizado o mal posicionado en la hendidura en una cirugía determinada.

Existió una predominancia de la hendidura del lado izquierdo $(71 \%)$ coincidiendo tanto con el trabajo de Navas-Aparicio (2007), como con los estudios de de Almeida et al. (2009) y el de Lucas et al. (2000).El diente canino superior temporal izquierdo (6.3) es el que se presenta más frecuentemente adyacente a la hendidura, tal como se observa en la Figura 1, y los dientes incisivos laterales temporales, son los que principalmente se localizan en la hendidura alveolar, sobresaliendo, el incisivo lateral superior izquierdo temporal con un $46,2 \%$.

A pesar de que los dientes temporales podrían presentar movilidad aumentada por la pronta erupción del diente permanente, y que los dientes adyacentes a la hendidura alveolar aún no habían recibido cirugía de injerto óseo que mejorara el soporte de hueso, no se encontró diferencia estadísticamente significativa en la movilidad dental para ninguno de los grupos de dientes analizados en relación con la hendidura o según su posición en boca (tipo de diente). Este resultado contrasta con lo reportado por Mutthineni et al. (2010) y Schultes et al., quienes encontraron un mayor grado de movilidad en los dientes permanentes asociados a hendiduras alveolares con cirugía de injerto óseo ya realizada.

La literatura reporta que, en pacientes con hendidura, el injerto óseo es importante en la prevención de problemas mucogingivales (Mutthineni et al.; de Almeida et al., 2012) y que los dientes en el área de la hendidura podrían tener mayor prevalencia de recesión gingival y disminución de encía queratinizada por la ausencia de soporte óseo (de Almeida et al., 2012). En el presente estudio únicamente un diente 6.3 , adyacente a la hendidura alveolar, presentó recesión gingival por vestibular (1 mm).

Existen estudios que demuestran que la cicatriz posterior a la cirugía en el área de la hendidura predispone a cambios marginales anatómicos (Costa et al.; de Almeida et al., 2009; Muthineni et al.), tales como pérdida de inserción y recesión gingival,especialmente en vestibular (de Almeida et al., 2012). En este estudio, los valores para encía queratinizada y ancho de la banda de encía insertada fueron significativamente mejores para los dientes no adyacentes a la hendidura alveolar en comparación con los otros dos grupos de estudio, condición que predispone al desarrollo tanto de gingivitis como de periodontitis.

Costa et al. en su estudio compararon la prevalencia de placa dental y gingivitis en niños de 5 a 6 años con dentición temporal y labio y paladar hendido versus niños sin hendiduras, sin encontrar diferencia estadísticamente significativa entre ambos grupos, por lo que concluyeron que el control de placa es inadecuado siempre en este rango de edad, con hendidura presente o sin ella, probablemente debido a la falta de destreza manual en los niños y la falta de colaboraciónde los padres durante el cepillado. En la presente investigación, el $47 \%$ de los dientes tenían placa dental, siendo significativamente mayor en los dientes que estaban dentro de la hendidura, posiblemente debido a que esta ubicación limita el acceso a la higiene oral. Además, la acumulación de placa predominó en vestibular (70 \%) en comparación a palatino (30 \%) indistintamente de la relación del diente con la hendidura alveolar,a pesar de ser las superficies vestibulares zonas más accesibles para la higiene oral, por lo que se sospecha que la lengua juega un papel fundamental en la lisis de la placa bacteriana palatina en este tipo de pacientes.

Diferentes estudios reportan una alta incidencia de formación de cálculo en pacientes con labio y paladar hendido en dientes relacionados con la hendidura alveolar (Schultes et al.; Boloor \& Thomas), lo cual no coincide con esta investigación donde el $100 \%$ de los dientes estaban libres de cálculo dental.

En cuanto a la profundidad de bolsa, se encuentra que las mayores profundidades se ubican en los dientes localizados dentro de la hendidura alveolar, alcanzando incluso mediciones tan severas como $11,5 \mathrm{~mm}$, siendo la medición máxima compatible con salud de 3 $\mathrm{mm}$. Al comparar los otros dos grupos de dientes adyacentes y no adyacentes a la hendidura alveolar, se en- 
cuentra que el primer grupo presenta profundidades de bolsa significativamente mayores que el segundo grupo. Resulta evidente que la ubicación de los dientes con respecto a la hendidura alveolar juega un rol fundamental en la profundidad de bolsa. Boloor \& Thomas encontraron que pacientes con labio y paladar hendido completo, y a quienes no se les había realizado la cirugía de injerto óseo, tenían una mayor incidencia de bolsas periodontales de más de 4 $\mathrm{mm}$, sangrado al sondaje y cálculo dental, siendo los dientes más afectados aquellos adyacentes a la hendidura alveolar. En contraste, Bastos et al. (2004) no encontraron bolsas periodontales en su grupo de estudio de 1 a 5 años, pero sí en pacientes de mayor edad, concluyendo que, a mayor edad, mayor prevalencia de bolsas periodontales (Mutthineni et al.).

Schultes et al. encontraron en su estudio un $100 \%$ de sangrado al sondaje en la región posterior y un $25 \%$ en la región anterior maxilar y mandibular, siendo los pacientes con labio y paladar hendido, aquellos con mayor incidencia de sangrado en el segmento anterior maxilar, específicamente en los dientes adyacentes a la hendidura (90\%). En la presente investigación, los dientes dentro de la hendidura alveolar fueron los que estadísticamente sangraron más al sondaje en comparación con los otros dos grupos de estudio. Si bien no existió diferencia estadísticamente significativa en cuanto al sangrado al sondaje entre los dientes adyacentes y no adyacentes a la hendidura alveolar, sí se encontró que los dientes adyacentes a la hendidura sangraron más que los dientes no adyacentes,sugiriendo un mayor grado de inflamación en esta zona asociado con la dificultad de higiene, y a otros factores etiológicos locales (posición inadecuada del diente, alteraciones a nivel de tejido blando, falta de adecuado soporte óseo, entre otros). Un hallazgo relevante a señalar es que un diente incisivo lateral temporal izquierdo adyacente a la hendidura alveolar y localizado hacia palatino, tenía una bolsa periodontal de $5 \mathrm{~mm}$ con sangrado y supuración.

Pocos dientes presentaron pérdida de inserción clínica. La mayor pérdida de inserción se registró en los dientes que se encontraban dentro de la hendidura alveolar. Estos datos contrastan con lo reportado por de Almeida et al. (2009), quienes manifestaron que la enfermedad periodontal determinada por la profundidad de bolsa y el nivel de inserción clínica en los dientes en el área de la hendidura no es mayor comparada con otras áreas.

En este estudio, se hallaron condiciones clínicas que pudieron afectar, el estado periodontal de los dien- tes temporales, como por ejemplo: pliegues de mucosa cicatrizales, tejido gingival asimétrico y con condiciones diferentes a las características normales del periodonto como consecuencia de cirugías realizadas, diente localizado en la hendidura alveolar, erupción incompleta del diente, mordida cruzada anterior, relación borde-borde anterior, incisivos superiores retroinclinados, mal alineamiento dental de los dientes temporales / discrepancia dento-alveolar, desgastes incisales de los dientes anteriores superiores, mordida abierta anterior por hábito de lengua protráctil, caries dental, resto radicular y colapso palatino de premaxila, muchos de ellos ya reportados en la literatura (Schultes et al.; Stec et al.; Lages et al., 2004; Perdikogianni et al., 2009; Boloor \& Thomas; de Almeida et al., 2012).

Aunque los pacientes de este estudio presentaban como antecedentes quirúrgicos comunes la plastía labial primaria y plastía de paladar primario, puede ser que alguna otra cirugía ocasionase alteraciones anatómicas locales que dificultaran la higiene oral y la aparición de problemas periodontales (por ejemplo: el cierre de fístulas palatinas y nasovestibulares).

Con respecto al análisis de las radiografías periapicales, se observó que predomina la hendidura alveolar completa en nueve pacientes. Hubo 7 casos, en los cuales no se pudo determinar el tipo de hendidura debido a la posición de los dientes adyacentes a ésta y a los dientes erupcionados en la hendidura alveolar. Es importante mencionar que no se estableció la altura de la cresta alveolar, ya que la toma de las radiografías no se pudo realizar con una técnica estándar por falta de completa cooperación del niño.

Es de relevancia mencionar, que un factor que puede ayudar a disminuir los problemas periodontales en la dentición adyacente a la hendidura alveolar es la realización del injerto óseo, pues se ha descrito que uno de los objetivos de éste a nivel de la hendidura alveolar no es solo dar continuidad al reborde alveolar, que se encuentra separado, sino proveer un desarrollo adecuado de hueso en la zona, para favorecer la erupción del canino y dar soporte periodontal a los dientes adyacentes a la hendidura alveolar (Bragger et al., 1985; AndlinSobocki et al., 1995; de Almeida et al., 2012).

\section{CONCLUSIÓN}

Los dientes más afectados, desde el punto de vista periodontal, en esta investigación, para la pobla- 
ción estudiada, son aquellos ubicados dentro de la hendidura alveolar, seguidos por los dientes adyacentes a la hendidura alveolar.

Un factor que puede ayudar a disminuir los problemas periodontales en la dentición adyacente a la hendidura alveolar es la realización del injerto óseo, cuyo objetivo es dar continuidad al reborde alveolar, proveer un desarrollo adecuado de hueso en la zona, para favorecer la erupción del canino y dar soporte periodontal a los dientes adyacentes a la hendidura alveolar , así como soporte a los demás tejidos de la cara.

Existe la necesidad de establecer un adecuado programa de mantenimiento periodontal en pacientes con labio y paladar hendido, por presentar mayor predisposición a problemas periodontales, así como la necesidad de fortalecer la educación sobre higiene oral en los padres o encargados de los niños.

\section{FUENTES DE FINANCIAMIENTO}

La presente investigación no ha recibido ayudas específicas provenientes de agencias del sector público, sector comercial o entidades sin ánimo de lucro.

NAVAS-APARICIO, M. C. \& VARGAS-MORA, M. A. Periodontal condition of primary teeth adjacent to the alveolar cleft in patients with non-syndromic unilateral cleft lip and palate, Hospital Nacional de Niños "Dr. Carlos Sáenz Herrera", Caja Costarricense de Seguro Social. Int. J. Odontostomat., 15(4):1009-1018, 2021.

ABSTRACT: The teeth adjacent to the alveolar cleft, in patients with cleft lip and palate, present a higher incidence of periodontal problems, according to the literature. The purpose of this study was to evaluate whether the temporary teeth adjacent to the alveolar cleft present a higher incidence of periodontal problems in children with non-syndromic complete unilateral cleft lip and palate, born in 2010 and 2011, treated at the Hospital Nacional de Niños "Dr. Carlos Sáenz Herrera", Caja Costarricense de Seguro Social. This prospective, cross-sectional, descriptive, and interventional study evaluated epidemiological, clinical and radiographic variables (bacterial plaque, pocket depth, insertion level, gingival recession, bleeding, keratinized and inserted gingiva, dental calculus and mobility, type of alveolar cleft). It used descriptive statistical analysis techniques frequency distribution, crossing of variables and construction of confidence intervals. Data analysis and statistical processing was performed in SPSS version 17.0 and in Excel. The group consisted of 17 patients, with an average age of 5 years. $47 \%$ dental plaque and $0 \%$ dental calculus were found. Pocket depth and bleeding were statistically higher for teeth in the alveolar cleft. The teeth adjacent to the cleft had less keratinized and inserted gingiva. Regarding dental mobility, there is no statistically significant difference. Only one tooth had gingival recession. The complete alveolar cleft predominated radiographically. Teeth close to the alveolar cleft have a greater predisposition to periodontal problems, so regular monitoring of periodontal health is relevant.

KEY WORDS: alveolar cleft, deciduous teeth, probing depth, clinical attachment level, periodontitis, gingivitis.

\section{REFERENCIA BIBLIOGRÁFICAS}

Aizpurua, E. A. Incidencia de labio y paladar hendido en una región de Venezuela. Período 1995-1999. Rev. Latinoam. Ortod. Odontopediatr., 2002:4, 2002. Disponible en: https:// www.ortodoncia.ws/publicaciones/2002/art-4/

American Academy of Periodontology. Glossary of Periodontal Terms. 4th ed. Michigan, American Academy of Periodontology, 2001.

Andlin-Sobocki, A.; Åke Eliasson, L. \& Paulin, G. Periodontal evaluation of teeth in bone grafted regions in patients with unilateral cleft lip and cleft palate. Am. J. Orthop., 107(2):144-52, 1995.

Boloor, V. \& Thomas, B. Comparison of periodontal status among patients with cleft lip, cleft palate, and cleft lip along with a cleft in palate and alveolus. J. Indian Soc. Periodontol., 14(3):168-72, 2010.

Bragger, U.; Schürch Jr., E.; Gusberti, F. A. \& Lang, N. P. Periodontal conditions in adolescents with cleft lip, alveolus and palate following treatment in a coordinated team approach. J. Clin. Periodontol., 12(6):494-502, 1985.

Costa, B.; de Oliveira, J. E.; Ribeiro Gomide, M. \& Pereira da Silva Rosa, O. Clinical and microbiological evaluation of the periodontal status of children with unilateral complete cleft lip and palate. Cleft Palate Craniofac. J., 40(6):585-9, 2003.

de Almeida, A. L.; Esper, L. A.; Amadei Pegoraro, T. \& do Valle, A. L. Gingival recession in individuals with cleft lip and palate: prevalence and severity. Cleft Palate Craniofac. J., 49(1):92-5, 2012.

de Almeida, A. L.; Gonzalez, M. K.; Greghi, S. L.; Conti, P. C. \& Pegoraro, L. F. Are teeth close to the cleft more susceptible to periodontal disease?. Cleft Palate Craniofac J. 46(2):161-5, 2009.

Gamba Garib, D.; Sayako Yatabe, M.; Okada Ozawa, T. \& da Silva Filho, O. G. Alveolar bone morphology in patients with bilateral complete cleft lip and palate in the mixed dentition: cone beam computed tomography evaluation. Cleft Palate Craniofac. J., 49(2):208-14, 2012.

Huynh-Ba, G.; Bräagger, U.; Zwahlen, M.; Lang, N. P. \& Salvi, G. E. Periodontal disease progression in subjects with orofacial clefts over a 25-year follow-up period. J. Clin. Periodontol., 36(10):83642, 2009.

Lages, E. M.; Marcos, B. \& Almeida Pordeus, I. Oral health of individuals with cleft lip, cleft palate or both. Cleft Palate Craniofac. J., 41(1):5963, 2004.

Lindhe, J.; Lang, N. P. \& Karring, T. Clinical Periodontology and Implant Dentistry. 5th ed. New York, Wiley, 2008.

Lucas, V. S.; Gupta, R.; Ololade, O.; Gelbier, M. \& Roberts, G. J. Dental health indices and caries associated microflora in children with unilateral cleft lip and palate. Cleft Palate Craniofac. J., 37(5):44752, 2000. 
NAVAS-APARICIO, M. C. \& VARGAS-MORA, M. A. Condición periodontal de los dientes temporales adyacentes a la hendidura alveolar en pacientes con labio y paladar hendido unilateral no sindrómico, Hospital Nacional de Niños "Dr. Carlos Sáenz Herrera”, Caja Costarricense de Seguro Social. Int. J. Odontostomat., 15(4):1009-1018, 2021.

Mutthineni, R. B.; Nutalapati, R. \& Kasagni, S. K. Comparison of oral hygiene and periodontal status in patients with cleft of palate and patients with unilateral cleft lip, palate and alveolus. J. Indian Soc. Periodontol., 14(4):235-40, 2010.

Navas-Aparicio, M. C. Crecimiento maxilar según severidad de hendidura labial, alveolar y palatina unilateral. Cir. Plast. Iberolatinoam., 38(4):349-57, 2012.

Navas-Aparicio, M. C. Incidencia de labio y paladar hendido en la población infantil costarricense en el periodo 1998-2004. Odontos, 9:32-8, 2007.

Newman, M. G.; Takei, H.; Carranza, F. A. \& Klokkevold, P. Clinical Periodontology. 9th ed. Philadelphia, Elsevier, 2002.

Perdikogianni, H.; Papaioannou, W.; Nakou, M.; Oulis, C. \& Papagiannoulis, L. Periodontal and microbiological parameters in children and adolescents with cleft lip and/or palate. Int. J. Paediatr. Dent., 19(6):455-67, 2009.

Salvi, G. E.; Bragger, U. \& Lang, N. P. Periodontal attachment loss over 14 years in cleft lip, alveolus and palate (CLAP, CL, CP) subjects not enrolled in a supportive periodontal therapy program. J. Clin. Periodontol., 30(9):840-5, 2003.

Schultes, G.; Gaggl, A. \& Kärcher, H. Comparison of periodontal disease in patients with clefts of palate and patients with unilateral clefts of lip, palate and alveolus. Cleft Palate Craniofac. J., 36(4):322-7, 1999

Stec, M.; Szczepan'ska, J.; Pypéc, J. \& Hirschfelder, U. Periodontal status and oral hygiene in two populations of cleft patient. Cleft Palate Craniofac. J., 44(1):73-8, 2007.

Uddin, N.; Molla, M. R.; Ahmed, M. U.; Bhuiyan, R. A.; Haider, I. A. \& Khairuzzaman, M. Evaluation of periodontal status and microorganisms in operated completes cleft-lip and palate patients. Int. J. Oral Maxillofac. Surg., 36(11):991, 2007.
Dirección para correspondencia:

María del Carmen Navas-Aparicio

Hospital Nacional de Niños «Carlos Sáenz Herrera»

Caja Costarricense de Seguro Social

Costa Rica 1654-1000

San José

COSTA RICA

Email:mcnavas@ccss.sa.cr 